ARTICLE

\title{
An antibonding valence band maximum enables defect-tolerant and stable GeSe photovoltaics
}

\author{
Shun-Chang Liu (1) 1,2,6, Chen-Min Dai ${ }^{3,6}$, Yimeng Min ${ }^{4}$, Yi Hou (i) ${ }^{4}$, Andrew H. Proppe (D) ${ }^{4}$, Ying Zhou (D) ${ }^{5}$,

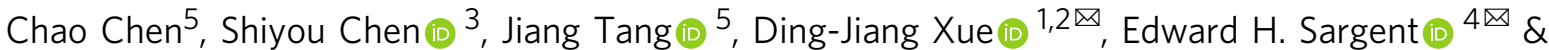 \\ Jin-Song Hu (i) ${ }^{1,2}$ 讹
}

In lead-halide perovskites, antibonding states at the valence band maximum (VBM)-the result of $\mathrm{Pb} 6 s-\mathrm{I} 5 p$ coupling-enable defect-tolerant properties; however, questions surrounding stability, and a reliance on lead, remain challenges for perovskite solar cells. Here, we report that binary GeSe has a perovskite-like antibonding VBM arising from Ge $4 s-\mathrm{Se} 4 p$ coupling; and that it exhibits similarly shallow bulk defects combined with high stability. We find that the deep defect density in bulk GeSe is $\sim 10^{12} \mathrm{~cm}^{-3}$. We devise therefore a surface passivation strategy, and find that the resulting GeSe solar cells achieve a certified power conversion efficiency of 5.2\%, 3.7 times higher than the best previously-reported GeSe photovoltaics. Unencapsulated devices show no efficiency loss after 12 months of storage in ambient conditions; 1100 hours under maximum power point tracking; a total ultraviolet irradiation dosage of $15 \mathrm{kWh} \mathrm{m}^{-2}$; and 60 thermal cycles from -40 to $85^{\circ} \mathrm{C}$.

\footnotetext{
${ }^{1}$ Beijing National Laboratory for Molecular Sciences (BNLMS), CAS Key Laboratory of Molecular Nanostructure and Nanotechnology, Institute of Chemistry, Chinese Academy of Sciences, Beijing 100190, China. ${ }^{2}$ University of Chinese Academy of Sciences, Beijing 100049, China. ${ }^{3}$ Key Laboratory of Polar Materials and Devices (MOE), East China Normal University, Shanghai 200241, China. ${ }^{4}$ Department of Electrical and Computer Engineering, University of Toronto, Toronto Ontario M5S 1A4, Canada. ${ }^{5}$ Wuhan National Laboratory for Optoelectronics (WNLO), Huazhong University of Science and Technology, Wuhan 430074, China. ${ }^{6} T h e s e$ authors contributed equally: Shun-Chang Liu, Chen-Min Dai. ${ }^{凶}$ email: djxue@iccas.ac.cn; ted.sargent@utoronto.ca; hujs@iccas.ac.cn
} 
$\mathrm{T}$ he macroscopic properties of a semiconductor depend on the chemical bonding between its constituent elements: electrostatics for ionic systems, and orbital hybridization for covalent systems. In an organic-inorganic hybrid perovskite solar cells (PSCs), power conversion efficiencies (PCEs) have increased from an initial $3.8 \%^{1}$ to a certified $25.2 \%^{2}$ in the last decade. The electronic structure of perovskites- $\mathrm{Pb} 6 s-\mathrm{I} 5 p$ antibonding states at the valence band maximum (VBM), contrasting with semiconductors such as GaAs and GaN that have a bonding VBM-is a key to the extraordinary performance of perovskite-based optoelectronics ${ }^{3-10}$. Because of this antibonding feature, defects in perovskites are confined to shallow states close to the band edges, instead of introducing states within the bandgap ${ }^{11-16}$.

Another bonding feature in perovskites is high ionicity, which enables thin-film fabrication, wherein perovskites form highly crystalline materials when prepared even at room temperature ${ }^{17,18}$. However, this high ionicity makes perovskites easily soluble in water and sensitive to moisture, inducing performance degradation ${ }^{19,20}$. Ionic perovskites also exhibit ion migration, a reason for $J-V$ hysteresis ${ }^{21-23}$.

We reasoned that the combination of strong covalent bonding, coupled with high stability and a perovskite-like antibonding VBM electronic structure, could potentially add new defecttolerant materials to photovoltaics.

We turned our attention to germanium monoselenide (GeSe), a material whose similar Pauling electronegativity of Ge (2.01) and Se (2.55) suggests a covalent semiconductor. GeSe has recently emerged as a promising absorber material for photovoltaics owing to its suitable bandgap $(\sim 1.14 \mathrm{eV})$, high absorption coefficient (greater than $\left.10^{5} \mathrm{~cm}^{-1}\right)$, high carrier mobility $(\sim 128$ $\mathrm{cm}^{2} \mathrm{~V}^{-1} \mathrm{~s}^{-1}$ ), and its earth-abundant, and $\mathrm{Pb}$-free composition $^{24-31}$. Its sublimation characteristic enables in-situ self purification of the raw material, leaving impurities in the sublimation source during film deposition ${ }^{24}$. Since GeSe (s) sublimes to GeSe (g) without decomposing into elemental species, it avoids the formation of undesired Ge and Se interstitials ${ }^{30}$. Whether or not GeSe can exhibit defect tolerance analogous to that of $\mathrm{Pb}$-based perovskites, especially in light of its perovskite-like $n s^{2}$ electronic configuration, has so far remained unclear.

We began by investigating defects in GeSe. We find that GeSe, which has a $4 s^{2}$ electronic configuration, possesses a perovskitelike antibonding VBM arising from Ge $4 s$-Se $4 p$ coupling; and that this leads to shallow bulk defects, and it also prevents GeSe oxidation. We then find that surface defects in GeSe photovoltaics have played a major role in device performance until now, and we, therefore, develop surface-passivated GeSe solar cells. These achieve a certified PCE of 5.2\%, surpassing the best previouslyreported GeSe results 3.7 fold. These devices exhibit excellent stability as required of thin-film photovoltaic modules (IEC 61646).

\section{Results}

Antibonding coupling for defect-tolerant GeSe. GeSe crystallizes in an orthorhombic layered structure with the Pnma 62 space group (Fig. 1a). Both Ge and Se atoms are three fold coordinated with each other. There is only one type of Ge and Se: GeSe is a binary chalcogenide both chemically and structurally. There are therefore only six possible point defects in GeSe: cation vacancy $\left(\mathrm{V}_{\mathrm{Ge}}\right)$, anion vacancy $\left(\mathrm{V}_{\mathrm{Se}}\right)$, cation interstitial $\left(\mathrm{Ge}_{\mathrm{i}}\right)$, anion interstitial $\left(\mathrm{Se}_{\mathrm{i}}\right)$, cation-replace-anion antisite $\left(\mathrm{Ge}_{\mathrm{Se}}\right)$, and anion-replace-cation antisite $\left(\mathrm{Se}_{\mathrm{Ge}}\right)$. This is simpler than in multicomponent semiconductors such as $\mathrm{Cu}(\mathrm{In}, \mathrm{Ga}) \mathrm{Se}_{2}$ (CIGS) and $\mathrm{Cu}_{2} \mathrm{ZnSn}(\mathrm{S}, \mathrm{Se})_{4}(\mathrm{CZTSSe})^{32,33}$.
We first used density functional theory (DFT) to calculate the bandstructure, density of states (DOS), and partial DOS of GeSe, since the electronic properties of point defects depend sensitively on the structure. The conduction band minimum (CBM) of GeSe is dominated by the Ge $4 p$ orbital, with significant coupling with the Se $4 p$ orbital; and negligible coupling with the Se $4 s$ orbital (Fig. 1b). This indicates the strong covalent character of GeSe, agreeing well with the above electronegativity analysis, and differing from perovskites with their high ionicity. As for the VBM, it is predominantly made up of the Se $4 p$ orbital and the Ge $4 p$ orbital due to $p-p$ coupling, with a substantial contribution from the Ge $4 s$ orbital. This is seen in the bandstructure of GeSe and the partial DOS of the Ge $4 s$ orbital (Fig. 1b).

The reason that the inner-shell Ge $4 s$ orbital is present in the VBM is illustrated through an atomic orbital picture (Fig. 1c): the Ge $4 s$ and $4 p$ orbitals are too far apart in energy to hybridize directly ${ }^{4,34}$; and the Se $4 p$ orbital is close to the Ge $4 s$ orbital in energy, allowing these to the couple and giving rise to a filled antibonding orbital at the $\mathrm{VBM}^{3,4}$. The $\mathrm{CBM}$ also has an antibonding character originating from the Ge $4 p-S e 4 p$ coupling. In the lone pair model, the asymmetrically layered-crystal structure of GeSe arising from the stereochemically active lone pairs accounts for the contribution of the Ge $4 s$ orbital to the $\mathrm{VBM}^{4}$. This differs from other IV-VI materials such as PbS and SnTe, which have symmetric structures ${ }^{4,7}$. The partial oxidation of $\mathrm{Ge}$ to its $\mathrm{Ge}^{2+}$ oxidation state contributes an antibonding $4 s$ character to the VBM, as in lead-halide perovskites.

We then calculated the formation energies and transition energy levels of the six possible point defects in GeSe mentioned above; we used the generalized gradient approximation (GGA) in these studies. The most striking observation is the high formation energies for all the defects, higher than $1.2 \mathrm{eV}$ in their neutral charge states (Fig. 1e, f). This is in contrast with $\mathrm{CH}_{3} \mathrm{NH}_{3} \mathrm{PbI}_{3}$, in which defects have low formation energies (close to zero) ${ }^{14,15}$ This is attributed to the stronger covalent Ge-Se bonds compared with the soft $\mathrm{Pb}-\mathrm{I}$ bonds in perovskites. The second notable feature is that $\mathrm{V}_{\mathrm{Ge}}$, with the lowest formation energy, has a shallow level with (-/0) and (2-/-) transition energy levels only 0.05 and $0.15 \mathrm{eV}$ above the VBM, whereas defects with deep levels such as $\mathrm{Ge}_{\mathrm{Se}}, \mathrm{Ge}_{\mathrm{i}}, \mathrm{V}_{\mathrm{Se}}$, and $\mathrm{Se}_{\mathrm{Ge}}$ have high formation energies (Supplementary Fig. 1). These are reconfirmed by Heyd-ScuseriaErnzerhof (HSE) calculations (Supplementary Fig. 2).

The low formation energy of $\mathrm{V}_{\mathrm{Ge}}$ is attributed to energetically unfavorable Ge $4 s-S e 4 p$ antibonding coupling, where the fully occupied antibonding state has no electronic energy ${ }^{14}$. The shallow nature of $\mathrm{V}_{\mathrm{Ge}}$ originates from the antibonding state at the VBM, a defect-tolerant electronic structure known to lead to shallow defects (Supplementary Fig. 3). This state pushes the VBM to a higher level such that the acceptor defect is shallower near the VBM than that without strong $s-p$ coupling. The strongly covalent GeSe with an antibonding VBM, therefore, exhibits mostly shallow defects.

We used deep-level transient spectroscopy (DLTS) to investigate the defect energy levels, concentrations, and types in semiconductor devices. The DLTS spectrum of GeSe photovoltaic devices fabricated using the previously-reported rapid thermal sublimation approach $^{24}$ is shown in Supplementary Fig. 4. Two positive peaks denoted as $\mathrm{H} 1$ and $\mathrm{H} 2$ are observed at $285 \mathrm{~K}$ and $310 \mathrm{~K}$, indicating two types of hole trap defects in the GeSe film. The activation energy $\left(E_{\mathrm{a}}\right)$ and capture cross-section $(\sigma)$ values determined from the Arrhenius plots are $0.35 \mathrm{eV}$ and $4.3 \times 10^{-23} \mathrm{~cm}^{2}$ in $\mathrm{H1}$, and $0.51 \mathrm{eV}$ and $7.6 \times 10^{-21} \mathrm{~cm}^{2}$ in $\mathrm{H} 2$, respectively (Fig. 1d). The concentration of defects $\left(N_{\mathrm{T}}\right)$ calculated from the equation of $N_{\mathrm{T}}=2 \Delta C^{*} N_{\mathrm{A}} / C_{0}\left(N_{\mathrm{A}}\right.$ is the net acceptor concentration in GeSe film) are $1.3 \times 10^{12} \mathrm{~cm}^{-3}$ for $\mathrm{H} 1$ and $3.0 \times 10^{12} \mathrm{~cm}^{-3}$ for $\mathrm{H} 2$, lower 
(a)

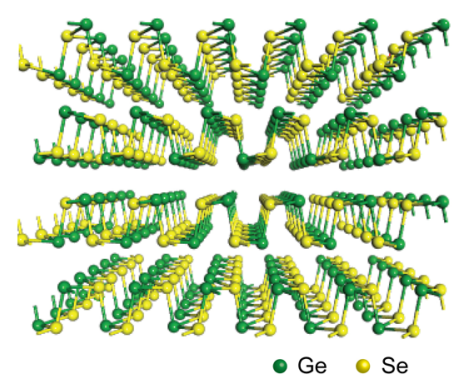

(c)

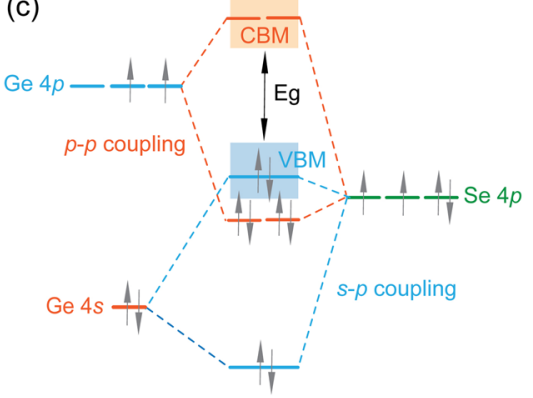

(d)

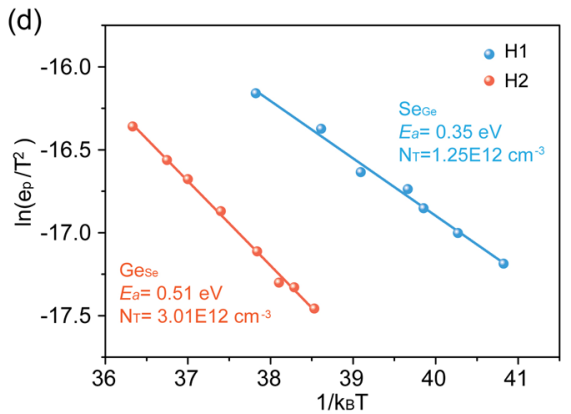

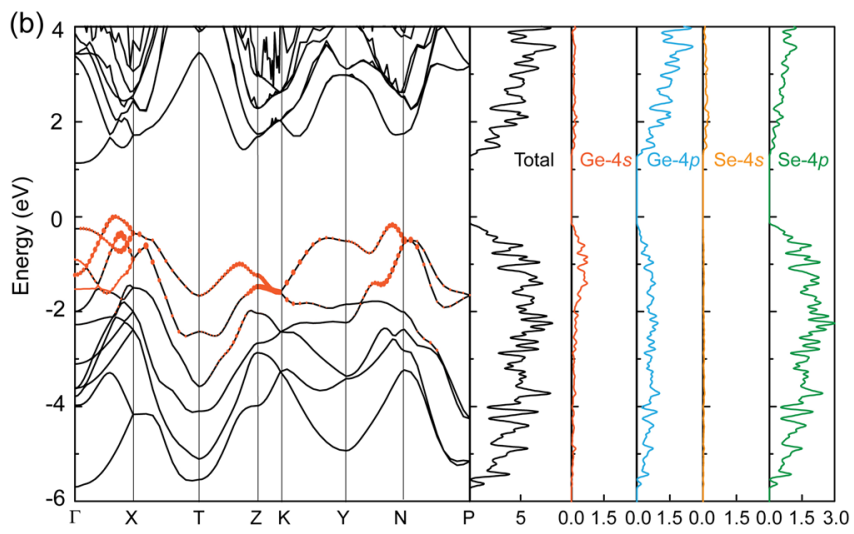
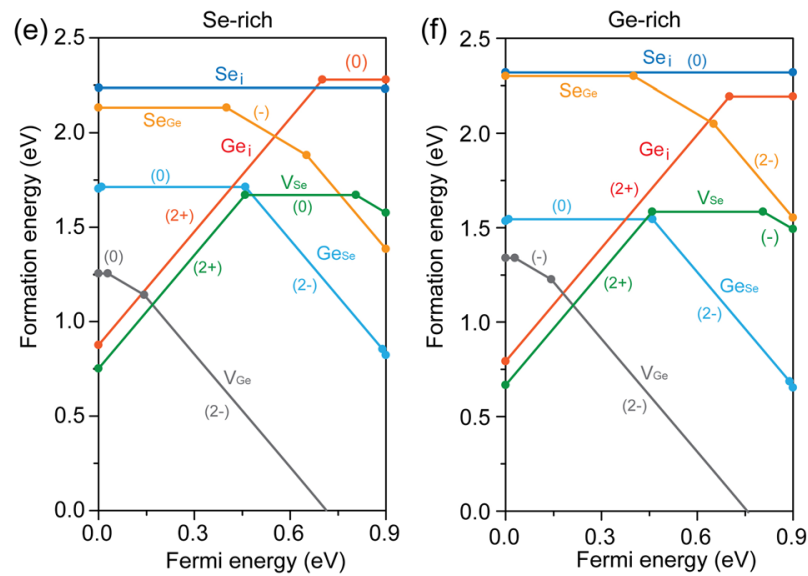

Fig. 1 Point defect properties of GeSe. a Crystal structure of GeSe. b Calculated bandstructure, density of states (DOS), and partial DOS projected on different elements of GeSe. c Schematic energy level diagram of the interactions in GeSe. d Arrhenius plots obtained from DLTS experiments. The solid lines represent the best fits to experimental data. Calculated formation energies of intrinsic point defects in GeSe under $\mathbf{e} \mathrm{Se}$-rich and $\mathbf{f} \mathrm{Ge}$-rich conditions as a function of the Fermi energy.

than state-of-art chalcogenides such as CIGS $\left(\sim 4.2 \times 10^{13} \mathrm{~cm}^{-3}\right)$ and CZTS $\left(\sim 3.7 \times 10^{14} \mathrm{~cm}^{-3}\right)^{35,36}$.

There are only two deep acceptor defects, $\mathrm{Se}_{\mathrm{Ge}}$ and $\mathrm{Ge}_{\mathrm{Se}}$. We associate the $\mathrm{H} 1$ and $\mathrm{H} 2$ defects observed in the DLTS fitting results at $0.35 \mathrm{eV}$ and $0.51 \mathrm{eV}$ with $\mathrm{Se}_{\mathrm{Ge}}$ and $\mathrm{Ge}_{\mathrm{Se}}$, respectively. No $\mathrm{V}_{\mathrm{Ge}}$ is observed in the DLTS measurement since $\mathrm{V}_{\mathrm{Ge}}$ is too shallow to produce a response in the DLTS signal. Note that the densities of deep defects in GeSe including $\mathrm{Se}_{\mathrm{Ge}}$ and $\mathrm{Ge}_{\mathrm{Se}}$ are at a magnitude of $10^{12} \mathrm{~cm}^{-3}$, well below the bulk density of GeSe $\left(\sim 10^{15}\right) \mathrm{cm}^{-3}$ dominated by $\mathrm{V}_{\mathrm{Ge}}{ }^{24,30}$. Admittance spectroscopy (AS) measurements further confirmed the low densities of deeplevel defects in GeSe. The $E_{\mathrm{a}}$ values deduced from the Arrhenius plots are $0.29 \mathrm{eV}$ and $0.45 \mathrm{eV}$, while the integrated defect densities of these two defects are $1.6 \times 10^{13} \mathrm{~cm}^{-3}$ and $3.5 \times 10^{12} \mathrm{~cm}^{-3}$ (Supplementary Fig. 5), respectively. In sum, defects with low formation energies generate only shallow levels, whereas deeplevel defects have high formation energies and their density is low.

Photovoltaic device performance. When we fabricated devices using an architecture of ITO/CdS/GeSe/Au, we obtained a low PCE of $1.4 \%$, with a $V_{\mathrm{oc}}$ of $0.23 \mathrm{~V}$, a $J_{\mathrm{sc}}$ of $15.7 \mathrm{~mA} \mathrm{~cm}^{-2}$, and a FF of $40 \%$ (Fig. 2a). We reasoned that this inferior performance could arise from surface states. We then characterized the density of interfacial defects at the CdS/GeSe heterojunction through a combination of capacitance-voltage $(\mathrm{C}-\mathrm{V})$ profiling and drivelevel capacitance profiling (DLCP) measurements. C-V measurements are sensitive to free carriers as well as bulk and interfacial defects, while DLCP measurements are responsive to free carriers and bulk defects ${ }^{37,38}$. Thus, the density of interfacial defects at the heterojunction is estimated by subtracting $N_{\text {DLCP }}$ (defect density calculated from DLCP) from $N_{\mathrm{C}-\mathrm{V}}$ (defect density calculated from $\mathrm{C}-\mathrm{V}$ ). We calculated an interfacial defect density of $2 \times 10^{12} \mathrm{~cm}^{-2}$ at the GeSe/CdS interface (Fig. 2b), which can lead to severe recombination losses.

We focused therefore on surface passivation of GeSe films. We posited that $\mathrm{Sb}_{2} \mathrm{Se}_{3}$ would act as a bridge between CdS and GeSe. Recently, a buried CdS/Sb $\mathrm{Se}_{3}$ homojunction has been reported to arise due to the interfacial diffusion of cadmium, forming a good interface between CdS and $\mathrm{Sb}_{2} \mathrm{Se}_{3}$ layers ${ }^{39}$. We applied DFT to investigate the interface formation energy between GeSe and $\mathrm{Sb}_{2} \mathrm{Se}_{3}$ with a preferred orientation of [111] for GeSe and [221] for $\mathrm{Sb}_{2} \mathrm{Se}_{3}$. The formation energy is $-0.12 \mathrm{eV}$, indicating that the growth of $\mathrm{GeSe}$ on $\mathrm{Sb}_{2} \mathrm{Se}_{3}$ is feasible. X-ray diffraction (XRD) was then used to characterize the orientation of both $\mathrm{GeSe}$ and $\mathrm{Sb}_{2} \mathrm{Se}_{3}$ layers. When we deposited a GeSe film onto a [211]-oriented $\mathrm{Sb}_{2} \mathrm{Se}_{3}$ layer (Supplementary Fig. 6), we found that the modified GeSe has a preferred [111] orientation, whereas the peaks of (200) and (400) with the lowest surface energies for GeSe disappear completely (Supplementary Fig. 7). This confirms the strong interaction between [211]-oriented $\mathrm{Sb}_{2} \mathrm{Se}_{3}$ and [111]-oriented $\mathrm{GeSe}$, in agreement with theoretical calculations.

Photovoltaic devices that use the modified GeSe films are improved with a $V_{\mathrm{oc}}$ of $0.36 \mathrm{~V}$, a $J_{\mathrm{sc}}$ of $26.9 \mathrm{~mA} \mathrm{~cm}^{-2}$, a FF of $54 \%$, and $\mathrm{aCE}$ of $5.2 \%$ (Fig. $2 \mathrm{a}$ ). This efficiency is $3.7 \times$ higher than that of control devices. DFT calculations were used to study further the role of $\mathrm{Sb}_{2} \mathrm{Se}_{3}$ : dangling bonds on the surface of [111]-oriented GeSe film lead to localized states inside the bandgap, causing recombination losses (Fig. 2c), whereas the electron distribution becomes delocalized following modification with $\mathrm{Sb}_{2} \mathrm{Se}_{3}$ (Fig. 2d). $\mathrm{C}-\mathrm{V}$ profiling and DLCP measurements reveal an order of magnitude lower interfacial defect density $\left(2 \times 10^{11} \mathrm{~cm}^{-2}\right)$ than 


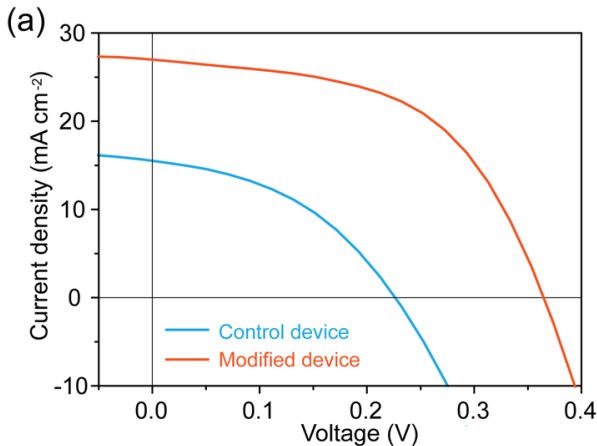

(c)

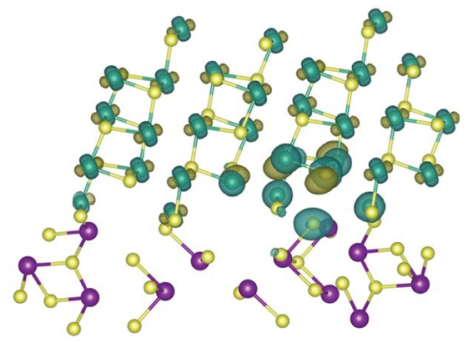

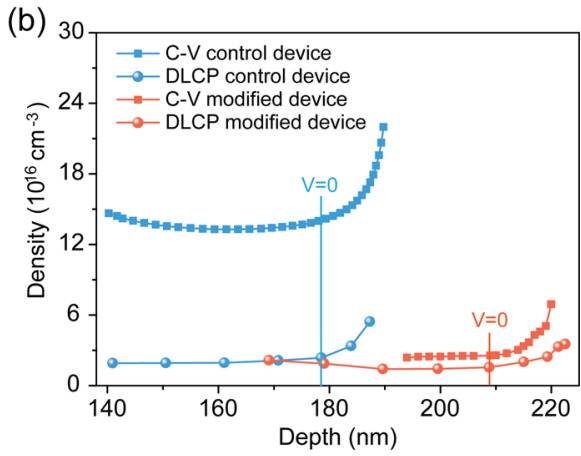

(d)

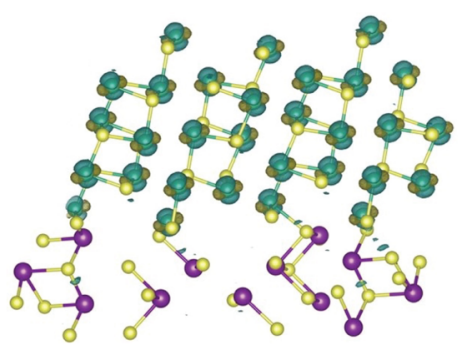

Fig. 2 Analysis of device performance. a J-V curves of control and modified GeSe devices. b C-V and DLCP characteristics of control and modified GeSe devices. DFT models for $\mathbf{c}$ trap like localized defects on the surface of GeSe film and $\mathbf{d}$ delocalized surface defects on GeSe after passivation.

(a)

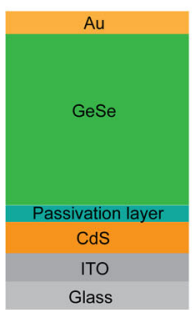

(b)

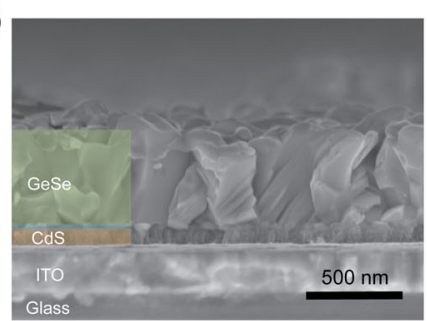

(c)

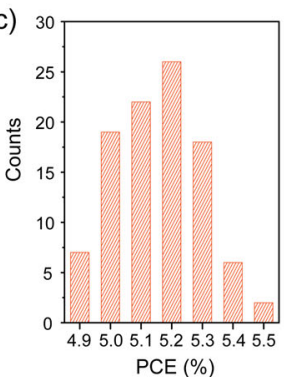

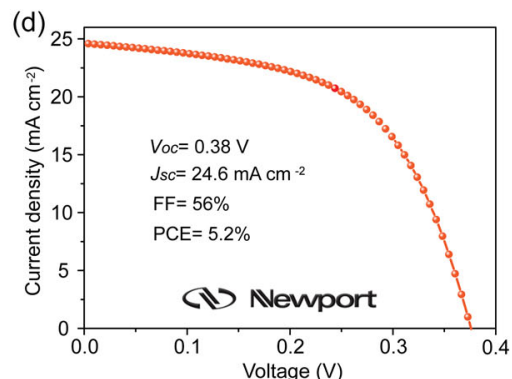
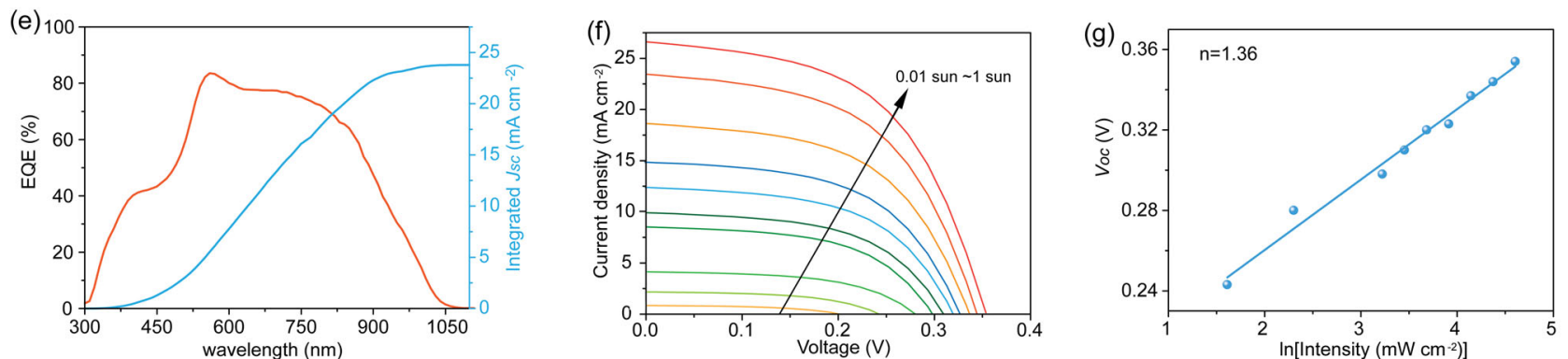

Fig. 3 Photovoltaic performance. a Schematic of GeSe thin-film solar cell architecture. b Cross-sectional SEM image of the GeSe device. c Histogram of device efficiencies obtained from 100 devices. $\mathbf{d} J-V$ curve and e EQE spectrum of the GeSe solar cell independently certified by Newport Corporation (Newport Corporation PV Laboratory, certificate \#1896). f J-V curves of a representative GeSe device measured under different intensities of simulated AM $1.5 \mathrm{G}$ illumination. $\mathbf{g}$ Light intensity-dependent $V_{\text {oc }}$ of GeSe solar cells. Neutral-density filters (THORLABS) were used to adjust the light intensity.

in the control devices $\left(2 \times 10^{12} \mathrm{~cm}^{-2}\right)$ (Fig. 2b). Achieving highperformance GeSe solar cells will require further work on the passivation of surface defects rather than bulk defects.

We fabricated over $100 \mathrm{GeSe}$ solar cells (device architecture in Fig. 3a). Figure $3 \mathrm{~b}$ shows a cross-sectional scanning electron microscope (SEM) image of a device; mapping with false coloring delineates the layers. The thickness of the GeSe layer is $500 \mathrm{~nm}$, and the thickness of the passivation layer is $10 \mathrm{~nm}$ (Supplementary Fig. 8). The average grain size of the GeSe film is $250 \mathrm{~nm}$ (Supplementary Fig. 9). There is a narrow distribution of PCE values (Fig. 3c), with an average efficiency of $5.2 \%$ and a standard deviation of $0.14 \%$. The best-performing device reaches a laboratory PCE of $5.5 \%\left(V_{\mathrm{oc}}=0.36 \mathrm{~V}, J_{\mathrm{sc}}=26.6 \mathrm{~mA} \mathrm{~cm}^{-2}\right.$, and $\mathrm{FF}=57 \%$ ) (Supplementary Fig. 10). No hysteresis is observed between forward and reverse scans.

We shipped an unencapsulated device to an accredited independent photovoltaic testing laboratory (Newport Corporation PV Lab, USA). This device displays a certified PCE of 5.2\% (Fig. 3d), with a corresponding $V_{\mathrm{oc}}$ of $0.38 \mathrm{~V}, J_{\mathrm{sc}}$ of $24.6 \mathrm{~mA} \mathrm{~cm}^{-2}$, and FF of $56 \%$ (accreditation certificate in Supplementary Fig. 11). This is the highest PCE reported so far for GeSe solar cells. Integration of the external quantum efficiency (EQE) collected 


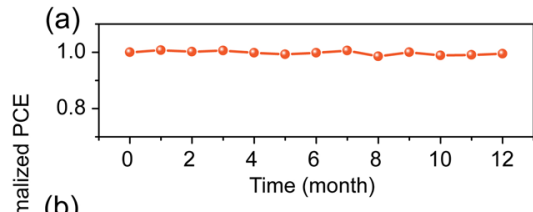

(b)

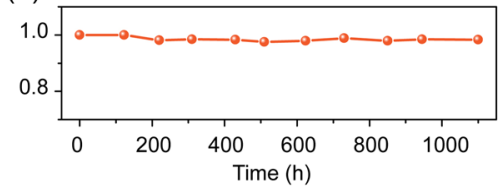

(c)

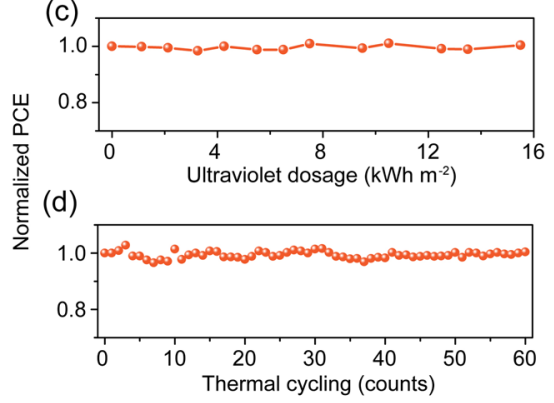

(e)

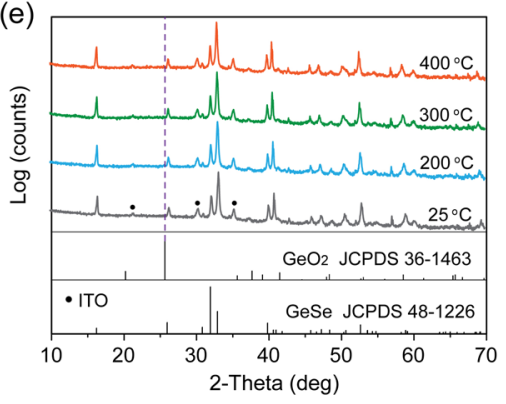

(g)

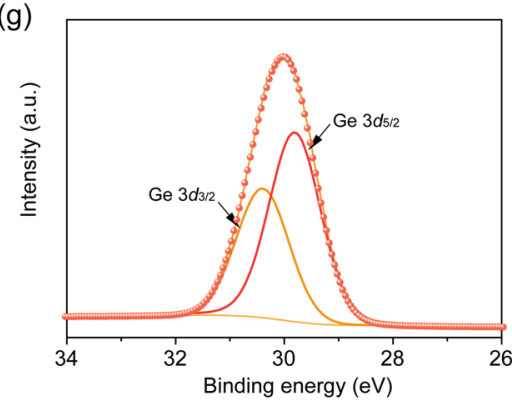

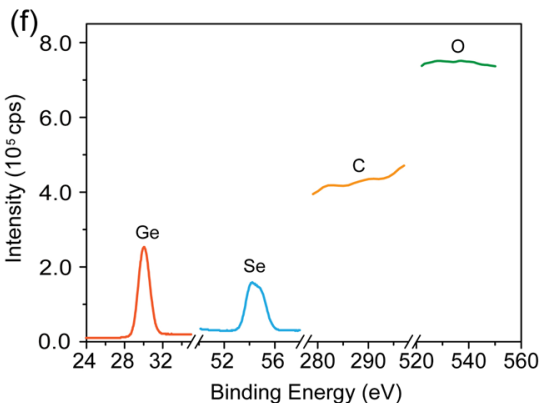

(h)

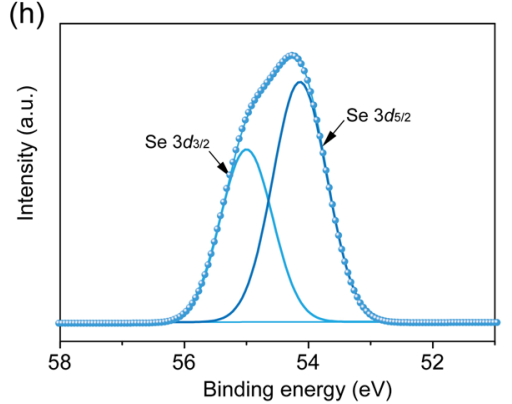

Fig. 4 Device and materials stability. a Long-term stability (ambient atmosphere, room temperature, relative humidity of 50-85\%), b operational stability (ambient atmosphere, continuous 1-sun illumination, close to maximum power point), c ultraviolet photostability (200-400 nm ultraviolet light irradiation), and $\mathbf{d}$ thermal cycling stability (cycling between -40 to $85^{\circ} \mathrm{C}$ for 60 cycles) of unencapsulated GeSe devices. e Temperature-dependent XRD patterns of GeSe film from 25 to $400^{\circ} \mathrm{C}$ in ambient atmosphere. XPS spectra of $\mathbf{f}$ Ge, Se, C, and O, $\mathbf{g}$ Ge $3 d$, and $\mathbf{h}$ Se $3 d$ in the GeSe film after temperature-dependent XRD measurements.

under the standard AM $1.5 \mathrm{G}$ solar spectrum yields a current density of $23.8 \mathrm{~mA} \mathrm{~cm}^{-2}$ (Fig. 3e), in good agreement with the $J_{\mathrm{sc}}$ value measured from $J-V$ characterization (within 5\% deviation) and also consistent with the absorption edge of GeSe (Supplementary Fig. 12). When we measured device performance at lowlight intensities (Fig. 3f), we found that devices exhibit PCE values of $5.3 \%, 6.3 \%$, and $8.6 \%$ (Supplementary Table 1 ) as we progress down to 0.01 sun. The corresponding light intensity-dependent $J_{\text {sc }}$ and $V_{\mathrm{oc}}$ are shown in Supplementary Fig. 13 and Fig. 3g. The power value a $(0.85)$ obtained from fitting to $J_{\text {sc }}$ measurement is close to unity (first-order); the slope obtained by linear fitting from $V_{\text {oc }}$ measurement is $1.36\left(\mathrm{k}_{\mathrm{B}} \mathrm{T} / \mathrm{q}\right)$, larger than $\mathrm{k}_{\mathrm{B}} \mathrm{T} / \mathrm{q}$ for trapfree solar cells, indicating that trap-assisted recombination is still present in these GeSe devices.

Device and materials stability. Stability of the GeSe devices was monitored by storing unencapsulated devices in an ambient atmosphere at room temperature and a relative humidity of $50-85 \%$. Devices retain $100 \%$ of their initial PCE after storage for 12 months (Fig. 4a). They also show negligible efficiency loss after continuous operation close to the maximum power point (MPP) under 1-sun illumination for $1100 \mathrm{~h}$ (Fig. 4b). We then investigated the ultraviolet photostability of unencapsulated devices under ultraviolet irradiation $(200-400 \mathrm{~nm})$. They retain their efficiency after exposure to an ultraviolet irradiation dosage of $15.5 \mathrm{kWh} \mathrm{m}^{-2}$ (Fig. 4c). The thermal stability was investigated by cycling the temperature from -40 to $85^{\circ} \mathrm{C}$ for a total of 60 cycles. They show no loss of efficiency after 60 thermal cycles (Fig. $4 \mathrm{~d}$ ).

Temperature-dependent XRD under an ambient atmosphere was applied to explore the origin of air and thermal stability. The film keeps its orthorhombic GeSe (JCPDS 48-1226) phase with no impurity peaks observed (such as $\mathrm{GeO}_{2}$ ) even up to $400{ }^{\circ} \mathrm{C}$ for 30 min (Fig. 4e). Since XRD is unable to detect amorphous components, we performed X-ray photoelectron spectroscopy (XPS) and energy-dispersive X-ray spectroscopy (EDS) on the same GeSe film after temperature-dependent XRD measurements. Oxygen and carbon are not detected in GeSe films (see magnified XPS spectrum at $520-550 \mathrm{eV}$ and $281-295$ $\mathrm{eV}$ ) (Fig. 4f), consistent with the EDS results (Supplementary Fig. 14). The Ge $3 d_{5 / 2}$ and $\mathrm{Ge} 3 d_{3 / 2}$ peaks in the Ge $3 d$ spectrum are observed at $29.85 \mathrm{eV}$ and $30.43 \mathrm{eV}$ (Fig. 4g), corresponding to $\mathrm{Ge}$ in the +2 oxidation state ${ }^{24}$. Gaussian-Lorentzian fitting confirms that no peak corresponding to +4 or 0 state of $\mathrm{Ge}$ is observed within the detection limit of the XPS instrument. The Se $3 d$ spectrum also reveals that $\mathrm{Se}$ is in the expected oxidation state of $\mathrm{Se}^{2-}$, corresponding to GeSe (Fig. $\left.4 \mathrm{f}\right)^{30}$. The above results, therefore, demonstrate the high air and thermal stability of GeSe. In addition, GeSe also exhibits excellent humidity and light stability (Supplementary Fig. 15). The $4 s^{2}$ electrons on the Ge cation in ionic perovskites are exposed, making them vulnerable to oxidation; while the lone-pair electrons on the $\mathrm{Ge}$ atoms in covalent GeSe participate in $\mathrm{Ge} 4 s-\mathrm{Se} 4 p$ coupling, leading to chemical inactivity.

\section{Discussion}

In summary, we report a binary and non-toxic photovoltaic absorber material, GeSe, with benign defect properties and high stability arising from its antibonding VBM from Ge $4 s-S e 4 p$ coupling. We found photovoltaic devices to be limited by surface defects rather than by bulk defects. By passivating these interfacial defects, we achieved a certified record PCE of 5.2\%. Unencapsulated GeSe devices exhibited no performance degradation under long-term ambient air, operating, ultraviolet soaking, and thermal cycling conditions. This work provides a deep understanding of the relationship between chemical bonding and macroscopic, device-relevant properties including the nature of defects and material's stability.

\section{Methods}

Solar cell fabrication. All devices were deposited on ITO (Sn-doped $\operatorname{In}_{2} \mathrm{O}_{3}$ ) conductive glass, which was cleaned using detergent, deionized water, acetone, and isopropanol in sequence. GeSe thin-film solar cells were fabricated with a structure 
consisting of CdS, passivated layer, GeSe, and Au. First, a CdS buffer layer was deposited by chemical bath deposition (CBD) on ITO conductive glass according to a previous report ${ }^{24}$. Then, the passivation layer $\left(\mathrm{Sb}_{2} \mathrm{Se}_{3}\right)$ was deposited using a modified rapid thermal evaporation (RTE) method ${ }^{40}$ : a tube furnace was set at $300{ }^{\circ} \mathrm{C}$ for $15 \mathrm{~min}$ to warm up the substrate, before raising the temperature to $550^{\circ} \mathrm{C}$ in $30 \mathrm{~s}$ to start the evaporation. We then kept to this temperature and carried out $2 \mathrm{~s}$ of deposition to obtain the $10 \mathrm{~nm}$ thick $\mathrm{Sb}_{2} \mathrm{Se}_{3}$ layer, then the power is turned off to stop the evaporation, and finally, the film was removed when it was cooled to $180^{\circ} \mathrm{C}$. We then transferred this $\mathrm{Sb}_{2} \mathrm{Se}_{3}$ modified substrate to another tube furnace in ambient air without protection; this next sept was to enable the deposition of GeSe film. GeSe films were fabricated by rapid thermal sublimation method as in a previous report ${ }^{24}$ : we preheated the GeSe powder and substrate at $350{ }^{\circ} \mathrm{C}$ for $20 \mathrm{~min}$, before quickly increasing the source temperature to $400{ }^{\circ} \mathrm{C}$ within $2 \mathrm{~s}$, maintaining this temperature for $5 \mathrm{~s}$, before finally turning off the heating. Finally, Au back contacts $(80 \mathrm{~nm})$ were deposited using a thermal evaporation system (Beijing Technol Science) through a shadow mask $\left(0.09 \mathrm{~cm}^{2}\right)$.

Materials characterization. Powder XRD patterns were recorded using a Rigaku D/Max-2500 diffractometer with a Cu target (Ka1 radiation, $\lambda=1.54056 \AA$ ). Highresolution XPS measurements were performed on an ESCALab220i-XL electron spectrometer (VG Scientific) using $300 \mathrm{~W} \mathrm{Al} \mathrm{Ka}$ radiation. The optical transmittance was measured using a UV - vis-near IR spectrophotometer (UH4150, HITACHI). Scanning electron microscopy (SEM) cross-sectional images were obtained by Hitachi S-4800 microscope. Atomic force microscopy (AFM) data were collected on a Bruker Dimension Icon microscope.

Device performance characterization. $J-V$ curves of the solar cells were obtained using an AM $1.5 \mathrm{G}$ solar simulator (Newport, USA) equipped with a Keithley 2420 source meter and $450 \mathrm{~W}$ xenon lamp (OSRAM) in the air at room temperature. Light intensity was adjusted using an NREL certified Si solar cell with a $\mathrm{KG}-2$ filter for approximating AM $1.5 \mathrm{G}$ light $\left(100 \mathrm{~mW} \mathrm{~cm}^{-2}\right)$. The device was covered with a metal mask with an aperture area of $0.09 \mathrm{~cm}^{2}$ during efficiency measurement. The $J-V$ curves were measured with a scanning rate of $100 \mathrm{mV} \mathrm{s}^{-1}$ (voltage step of $20 \mathrm{mV}$ and delay time of $200 \mathrm{~ms}$ ). Both forward (-1 to $1 \mathrm{~V}$ ) and backward $(1$ to $-1 \mathrm{~V})$ scans were recorded.

Electrical characterization. DLTS measurements were performed using an FT1030 HERA DLTS system equipped with a JANIS VPF-800 cryostat controller on the high-performance device. The temperature was scanned between 140 and $380 \mathrm{~K}$. The reverse bias voltage was set to $-0.5 \mathrm{~V}$. The filling pulse voltage and width were $0.4 \mathrm{~V}$ and $20 \mathrm{~ms}$, respectively. The C-V curves and DLCP spectra were obtained using a Keithley 4200. C-V measurements were performed at room temperature in an electromagnetic shielding box at a frequency of $10 \mathrm{kHz}$ and an A.C. amplitude of $30 \mathrm{mV}$. The D.C. bias voltage was scanned from $-1.0 \mathrm{~V}$ to $0.5 \mathrm{~V}$ with a step size of $0.01 \mathrm{~V}$. DLCP measurements were performed with an A.C. amplitude ranging from $14 \mathrm{mV}$ to $140 \mathrm{~V}$ and D.C. bias voltage from $-0.2 \mathrm{~V}$ to $0.2 \mathrm{~V}$.

Light-soaking test. Devices were illuminated using a Xe light source (PLS-SXE300, 1.35 sun intensity) with an AM 1.5 light filter. A $160 \Omega$ resistor was connected and the load was measured to be $175 \Omega$ due to the contribution from the additional connection circuit. The device was continuously operated near the MPP, and the measured output current density was $\sim 32 \mathrm{~mA} \mathrm{~cm}^{-2}$. The temperature of the device was maintained at $45-55^{\circ} \mathrm{C}$ without any external cooling. Every $100 \mathrm{~h}$, the device was subjected to the standard AM 1.5 to collect $J-V$ curves as described above after cooling down to room temperature within a few minutes.

Ultraviolet stability tests. Devices were exposed to UV light with wavelength range from 200 to $400 \mathrm{~nm}$ (Xe light source, PLS-SXE300) for $62 \mathrm{~h}$ and the power was kept at $25 \mathrm{~mW} \mathrm{~cm}^{-2}$. The devices were put on a hot plate maintaining the temperature at $60^{\circ} \mathrm{C}$. After illumination, we took out the device and measured the $J$ - $V$ curves under AM 1.5 illumination. Please note that compared to the IEC 61646 protocol, our measurement included a deeper range of ultraviolet light (for the 200-280 nm components)

Thermal cycling test. The devices were placed inside a high-low temperature test chamber under the atmosphere. Chamber temperature was periodically changed from -40 to $85^{\circ} \mathrm{C}$ for 60 cycles. For every cycle, the total time is $70 \mathrm{~min}$ and the device temperature remained stable at each extreme for $10 \mathrm{~min}$. After each cycle, the device temperature was adjusted to $298 \mathrm{~K}$ and device performance was measured.

Density functional theory. The crystal structure, total energy, and bandstructure were calculated using DFT methods as implemented in the Vienna ab initio simulation package (VASP) code $^{41}$. Frozen-core projector augmented-wave (PAW) pseudopotentials and a plane wave basis set with an energy cutoff of $520 \mathrm{eV}$ were employed, with a $8 \times 8 \times 4$ Monkhorst-Pack k-point mesh included in the Brillouin zone integration for the 16 -atom primitive cell and a $3 \times 3 \times 3$ mesh for the 64 - atom supercell, which was used for the calculation of defect properties ${ }^{32}$. Test calculations were performed with a higher energy cutoff, denser k-point mesh, and larger supercell size, and we found the same trends. The GGA to the exchangecorrelation functional was used in all the calculations, which predicted a bandgap around $0.9 \mathrm{eV}$ for $\mathrm{GeSe}$, slightly lower than the experimental value at $1.1 \mathrm{eV}$. To estimate the influence of GGA on the calculated results, the non-local hybrid functional was also used which predicted the bandgap more accurately ${ }^{42}$, showing that the conclusions were not influenced by the specific functionals.

Reporting Summary. Further information on research design is available in the Nature Research Reporting Summary linked to this article.

\section{Data availability}

The data that support the findings of this study are available on reasonable request from the corresponding author.

Received: 2 May 2020; Accepted: 4 January 2021; Published online: 28 January 2021

\section{References}

1. Kojima, A., Teshima, K., Shirai, Y. \& Miyasaka, T. Organometal halide perovskites as visible-light sensitizers for photovoltaic cells. J. Am. Chem. Soc 131, 6050-6051 (2009).

2. National Renewable Energy Laboratory. Best Research-Cell Efficiencies https://www.nrel.gov/pv/assets/pdfs/best-research-cell-efficiencies.20200406.pdf.

3. Walsh, A. \& Zunger, A. Instilling defect tolerance in new compounds. Nat. Mater. 16, 964-967 (2017).

4. Walsh, A., Payne, D. J., Egdell, R. G. \& Watson, G. W. Stereochemistry of post-transition metal oxides: revision of the classical lone pair model. Chem. Soc. Rev. 40, 4455-4463 (2011).

5. Kurchin, R. C., Gorai, P., Buonassisi, T. \& Stevanović, V. Structural and chemical features giving rise to defect tolerance of binary semiconductors. Chem. Mater. 30, 5583-5592 (2018).

6. Brandt, R. E., Stevanović, V., Ginley, D. S. \& Buonassisi, T. Identifying defecttolerant semiconductors with high minority-carrier lifetimes: beyond hybrid lead halide perovskites. MRS Commun. 5, 265-275 (2015).

7. Ganose, A. M., Savory, C. N. \& Scanlon, D. O. Beyond methylammonium lead iodide: prospects for the emergent field of $\mathrm{ns}^{2}$ containing solar absorbers. Chem. Commun. 53, 20-44 (2017).

8. Xiao, Z., Meng, W., Wang, J., Mitzi, D. B. \& Yan, Y. Searching for promising new perovskite-based photovoltaic absorbers: the importance of electronic dimensionality. Mater. Horiz. 4, 206-216 (2017).

9. Zakutayev, A. et al. Defect tolerant semiconductors for solar energy conversion. J. Phys. Chem. Lett. 5, 1117-1125 (2014).

10. Pandey, M. et al. Defect-tolerant monolayer transition metal dichalcogenides. Nano Lett. 16, 2234-2239 (2016).

11. Maughan, A. E. et al. Defect tolerance to intolerance in the vacancy-ordered double perovskite semiconductors $\mathrm{Cs}_{2} \mathrm{SnI}_{6}$ and $\mathrm{Cs}_{2} \mathrm{TeI}_{6}$. J. Am. Chem. Soc. 138, 8453-8464 (2016).

12. Ball, J. M. \& Petrozza, A. Defects in perovskite-halides and their effects in solar cells. Nat. Energy 1, 16149 (2016).

13. Huang, J., Yuan, Y., Shao, Y. \& Yan, Y. Understanding the physical properties of hybrid perovskites for photovoltaic applications. Nat. Rev. Mater. 2, 17042 (2017).

14. Yin, W.-J., Shi, T. \& Yan, Y. Unusual defect physics in $\mathrm{CH}_{3} \mathrm{NH}_{3} \mathrm{PbI}_{3}$ perovskite solar cell absorber. Appl. Phys. Lett. 104, 063903 (2014).

15. Xiao, Z., Song, Z. \& Yan, Y. From lead halide perovskites to lead-free metal halide perovskites and perovskite derivatives. Adv. Mater. 31, 1803792 (2019).

16. Steirer, K. X. et al. Defect tolerance in methylammonium lead triiodide perovskite. ACS Energy Lett. 1, 360-366 (2016).

17. Xue, D.-J. et al. Regulating strain in perovskite thin films through chargetransport layers. Nat. Commun. 11, 1514 (2020).

18. Liu, D. \& Kelly, T. L. Perovskite solar cells with a planar heterojunction structure prepared using room-temperature solution processing techniques. Nat. Photonics 8, 133 (2013).

19. Boyd, C. C., Cheacharoen, R., Leijtens, T. \& McGehee, M. D. Understanding degradation mechanisms and improving stability of perovskite photovoltaics. Chem. Rev. 119, 3418-3451 (2019).

20. Jiang, Q. et al. Surface passivation of perovskite film for efficient solar cells. Nat. Photonics 13, 460-466 (2019).

21. Eames, C. et al. Ionic transport in hybrid lead iodide perovskite solar cells. Nat. Commun. 6, 7497 (2015).

22. Hoke, E. T. et al. Reversible photo-induced trap formation in mixed-halide hybrid perovskites for photovoltaics. Chem. Sci. 6, 613-617 (2015). 
23. Zhao, Y.-C. et al. Quantification of light-enhanced ionic transport in lead iodide perovskite thin films and its solar cell applications. Light Sci. Appl. 6, e16243-e16243 (2017).

24. Xue, D.-J. et al. GeSe thin-film solar cells fabricated by self-regulated rapid thermal sublimation. J. Am. Chem. Soc. 139, 958-965 (2017).

25. Wang, X. et al. Short-wave near-infrared linear dichroism of two-dimensional germanium selenide. J. Am. Chem. Soc. 139, 14976-14982 (2017)

26. Liu, S.-C. et al. Investigation of physical and electronic properties of GeSe for photovoltaic applications. Adv. Electron. Mater. 3, 1700141 (2017).

27. Zhou, X. et al. Highly anisotropic GeSe nanosheets for phototransistors with ultrahigh photoresponsivity. Adv. Sci. 5, 1800478 (2018).

28. Yang, Y. et al. In-plane optical anisotropy of low-symmetry 2D GeSe. Adv. Opt. Mater. 7, 1801311 (2019).

29. Yang, Z. et al. WSe $2 / G e S e$ heterojunction photodiode with giant gate tunability. Nano Energy 49, 103-108 (2018).

30. Liu, S.-C., Yang, Y., Li, Z., Xue, D.-J. \& Hu, J.-S. GeSe thin-film solar cells. Mater. Chem. Front. 4, 775-787 (2020).

31. Murgatroyd, P. A. E. et al. GeSe: optical spectroscopy and theoretical study of a van der Waals solar absorber. Chem. Mater. 32, 3245-3253 (2020).

32. Walsh, A., Chen, S., Wei, S.-H. \& Gong, X.-G. Kesterite thin-film solar cells: advances in materials modelling of $\mathrm{Cu}_{2} \mathrm{ZnSnS}_{4}$. Adv. Energy Mater. 2, 400-409 (2012).

33. Chen, S., Walsh, A., Gong, X.-G. \& Wei, S.-H. Classification of lattice defects in the kesterite $\mathrm{Cu}_{2} \mathrm{ZnSnS}_{4}$ and $\mathrm{Cu}_{2} \mathrm{ZnSnSe}_{4}$ earth-abundant solar cell absorbers. Adv. Mater. 25, 1522-1539 (2013).

34. Waghmare, U. V., Spaldin, N. A., Kandpal, H. C. \& Seshadri, R. Firstprinciples indicators of metallicity and cation off-centricity in the IV-VI rocksalt chalcogenides of divalent Ge, Sn, and Pb. Phys. Rev. B 67, 125111 (2003).

35. Qi, Y.-F. et al. Engineering of interface band bending and defects elimination via a Ag-graded active layer for efficient $(\mathrm{Cu}, \mathrm{Ag})_{2} \mathrm{ZnSn}(\mathrm{S}, \mathrm{Se})_{4}$ solar cells. Energy Environ. Sci. 10, 2401-2410 (2017).

36. Repins, I. L. et al. Comparison of device performance and measured transport parameters in widely-varying $\mathrm{Cu}(\mathrm{In}, \mathrm{Ga})(\mathrm{Se}, \mathrm{S})$ solar cells. Prog. Photovolt. 14, 25-43 (2006).

37. Wang, W. et al. Device characteristics of CZTSSe thin-film solar cells with 12.6\% efficiency. Adv. Energy Mater. 4, 1301465 (2014).

38. Duan, H.-S. et al. The role of sulfur in solution-processed $\mathrm{Cu}_{2} \mathrm{ZnSn}(\mathrm{S}, \mathrm{Se})_{4}$ and its effect on defect properties. Adv. Funct. Mater. 23, 1466-1471 (2013).

39. Zhou, Y. et al. Buried homojunction in $\mathrm{CdS} / \mathrm{Sb}_{2} \mathrm{Se}_{3}$ thin film photovoltaics generated by interfacial diffusion. Appl. Phys. Lett. 111, 013901 (2017).

40. Zhou, Y. et al. Thin-film $\mathrm{Sb}_{2} \mathrm{Se}_{3}$ photovoltaics with oriented one-dimensional ribbons and benign grain boundaries. Nat. Photonics 9, 409-415 (2015).

41. Kresse, G. \& Furthmüller, J. Efficient iterative schemes for ab initio totalenergy calculations using a plane-wave basis set. Phys. Rev. B 54,11169-11186 (1996).

42. Heyd, J. \& Scuseria, G. E. Efficient hybrid density functional calculations in solids: assessment of the Heyd-Scuseria-Ernzerhof screened Coulomb hybrid functional. J. Chem. Phys. 121, 1187-1192 (2004).

\section{Acknowledgements}

This work is supported by the National Natural Science Foundation of China (21922512, 21875264, 61725401), the Youth Innovation Promotion Association CAS (2017050). The work of Y.M., Y.H., A.P., and E.H.S. is supported by the US Department of the Navy, Office of Naval Research (Grant Award NO. N00014-17-1-2524).

\section{Author contributions}

D.-J.X. conceived the idea and designed the experiments. S.-C.L. prepared films, fabricated devices, and characterized them. C.-M.D., Y.M., and S.C. performed the DFT calculations and analyzed the results. Y.Z. and C.C. assisted in the device characterization. Y.H. and A.P. helped with the manuscript preparation. J.T. discussed the results and commented on the paper. D.-J.X., S.-C.L., and E.H.S. wrote the paper. E.H.S. supervised the manuscript preparation. D.-J.X. and J.-S H. supervised the project. All authors read and commented on the manuscript.

\section{Competing interests}

The authors declare no competing interests.

\section{Additional information}

Supplementary information The online version contains supplementary material available at https://doi.org/10.1038/s41467-021-20955-5.

Correspondence and requests for materials should be addressed to D.-J.X., E.H.S. or J.-S.H.

Peer review information Nature Communications thanks the anonymous reviewer(s) for their contribution to the peer review of this work. Peer reviewer reports are available.

Reprints and permission information is available at http://www.nature.com/reprints

Publisher's note Springer Nature remains neutral with regard to jurisdictional claims in published maps and institutional affiliations.

Open Access This article is licensed under a Creative Commons Attribution 4.0 International License, which permits use, sharing, adaptation, distribution and reproduction in any medium or format, as long as you give appropriate credit to the original author(s) and the source, provide a link to the Creative Commons license, and indicate if changes were made. The images or other third party material in this article are included in the article's Creative Commons license, unless indicated otherwise in a credit line to the material. If material is not included in the article's Creative Commons license and your intended use is not permitted by statutory regulation or exceeds the permitted use, you will need to obtain permission directly from the copyright holder. To view a copy of this license, visit http://creativecommons.org/ licenses/by/4.0/

(C) The Author(s) 2021 